\title{
A NETWORK OF CONGRUENCES ON AN INVERSE SEMIGROUP'
}

\author{
BY
}

\author{
MARIO PETRICH AND NORMAN R. REILLY
}

\begin{abstract}
A congruence $\rho$ on an inverse semigroup $S$ is determined uniquely by its kernel and its trace. Denoting by $\rho^{\mathrm{min}}$ and $\rho_{\min }$ the least congruence on $S$ having the same kernel and the same trace as $\rho$, respectively, and denoting by $\omega$ the universal congruence on $S$, we consider the sequence $\omega, \omega^{\min }, \omega_{\min },\left(\omega^{\min }\right)_{\min },\left(\omega_{\min }\right)^{\min } \ldots$. These congruences, together with the intersections of corresponding pairs, form a sublattice of the lattice of all congruences on $S$. We study the properties of these congruences and establish several properties of the quasivarieties of inverse semigroups induced by them.
\end{abstract}

1. Introduction. Congruences on inverse semigroups deserve a careful study for several reasons. To start with, congruences on any semigroup provide some information about its homomorphic images, thereby occasionally illuminating the structure of the semigroup itself. Secondly, some of the most important structure theorems for inverse semigroups are based on the knowledge or a simple characterization of some special congruences on the semigroup, notably the least group or semilattice congruence. Thirdly, the study of various types of congruences on a general inverse semigroup provides a certain amount of information about their possible structure as well as making it possible to single out various classes of inverse semigroups of particular interest. Finally, a determination of all congruences on some inverse semigroups of known structure provides further insight into their nature.

The present study consists of an initial investigation of properties of congruences on an arbitrary inverse semigroup which are obtained by a recursive process based on the concepts of the kernel and the trace of a congruence. Specifically, we proceed as follows. Let $S$ be an inverse semigroup and $\rho$ be a congruence on $S$. Then $\operatorname{ker} \rho$, the kernel of $\rho$, is the set of all elements of $S \rho$-related to idempotents, and $\operatorname{tr} \rho$, the trace of $\rho$, is the restriction of $\rho$ to the idempotents of $S$. We denote by $\rho^{\min }$ the least congruence on $S$ having the same kernel as $\rho$, and by $\rho_{\min }$ the least congruence on $S$ having the same trace as $\rho$. Starting with the universal congruence $\omega$ on $S$, we form two sequences:

$$
\begin{aligned}
& \omega \supseteq \omega_{\min } \supseteq\left(\omega_{\min }\right)^{\min } \supseteq\left(\left(\omega_{\min }\right)^{\min }\right)_{\min } \supseteq \ldots, \\
& \omega \supseteq \omega^{\min } \supseteq\left(\omega^{\min }\right)_{\min } \supseteq\left(\left(\omega^{\min }\right)_{\min }\right)^{\min } \supseteq \ldots,
\end{aligned}
$$

Received by the editors December 10, 1979 and, in revised form, February 18, 1981.

1980 Mathematics Subject Classification. Primary 20M10.

Key words and phrases. Inverse semigroups, congruences, lattice of congruences, implications, kernel and trace of a congruence.

'This work was supported, in part, by NRC Grant A4044. 
These congruences, together with the intersections $\omega_{\min } \cap \omega^{\min },\left(\omega_{\min }\right)^{\min } \cap$ $\left(\omega^{\mathrm{min}}\right)_{\min }, \ldots$, form a sublattice of the lattice of all congruences on $S$.

We study this sublattice, in considerable detail. The quotients $\left\{S / \omega_{\min }\right\}$, $\left\{S / \omega^{\min }\right\}, \ldots$, as $S$ runs over all inverse semigroups, form quasivarieties, some of which are well known. Indeed, $\omega_{\min }=\sigma, \omega^{\min }=\eta,\left(\omega_{\min }\right)^{\min }=\pi,\left(\omega^{\min }\right)_{\min }=\nu$, $\left(\left(\omega^{\min }\right)_{\min }\right)^{\min }=\delta$ are, respectively, the least group, semilattice, $E$-unitary, semilattice of groups, and strongly $E$-reflexive congruence on $S$. We thus are able to include these well-known and remarkable congruences on $S$ into a system which, of course, continues indefinitely.

It is hoped that further properties of the min network can be established, for example, a simple system of implications for the resulting quasivarieties. For we have the feeling that what we have achieved is only the tip of an iceberg. One can analogously consider the sequence of congruences which start with the identical relation and recursively take $\rho^{\max }$ and $\rho_{\max }$ defined as the greatest congruence on $S$ having the same kernel or trace, respectively, as $\rho$.

2. Background. Throughout $S$ denotes an arbitrary inverse semigroup with the semilatice $E$ of idempotents.

We will adopt the notation and terminology of Howie [2], to which the reader is referred for basic information and results on inverse semigroups. For an arbitrary inverse semigroup $T$, we denote by $E_{T}$ the semilattice of its idempotents. A congruence $\tau$ on $E$ is a normal congruence if

$$
e \tau f \Rightarrow a^{-1} e a \tau a^{-1} f a \quad(e, f \in E, a \in S) .
$$

It was observed by Reilly and Scheiblich [10] that the restriction of any congruence $\rho$ on $S$ to $E$ is a normal congruence on $E$ called (see Petrich [8]) the trace of $\rho$ and denoted by $\operatorname{tr} \rho$. It was shown in [10] that the relation $\theta$ on the lattice $\Lambda(S)$ of congruences on $S$ given by

$$
\rho \theta \tau \Leftrightarrow \operatorname{tr} \rho=\operatorname{tr} \tau
$$

is a congruence such that each class is a complete modular sublattice of $\Lambda(S)$.

LeMma 2.1 [10, TheOREM 6.2]. Let $\tau$ be a normal congruence on $E$. Then the relation $\sigma_{\tau}$ defined on $S$ by

$$
a \sigma_{\tau} b \Leftrightarrow a e=b e, \text { for some } e \in E, e \tau a^{-1} a \tau b^{-1} b
$$

is the smallest congruence on $S$ such that $\operatorname{tr} \sigma_{\tau}=\tau$.

For any congruence $\rho$ on $S$, Scheiblich [11] defined the kernel of $\rho$, which we will denote by $\operatorname{ker} \rho$, to be

$$
\operatorname{ker} \rho=\left\{a \in S \mid a \rho e \text { for some } e \in E_{S}\right\}
$$

and showed how $\rho$ can be reconstructed from its kernel and trace.

Lemma 2.2 [11, TheOREM 2.1]. Let $\rho$ be a congruence on $S$. Then

$$
a \rho b \Leftrightarrow a^{-1} a \operatorname{tr} \rho b^{-1} b \text { and } a b^{-1} \in \operatorname{ker} \rho \text {. }
$$


Green [1, Theorems 3.3,3.4] studied the mapping $\rho \rightarrow \operatorname{ker} \rho$ and, among other things, proved that for each congruence $\rho$ on $S$, there exists a minimum congruence on $S$ having the same kernel as $\rho$.

Notation 2.3. For any congruence $\rho$ on $S$, we denote by $\rho^{\min }$ and $\rho_{\min }$ the minimum congruence $\theta$ on $S$ such that $\operatorname{ker} \theta=\operatorname{ker} \rho$ and $\operatorname{tr} \theta=\operatorname{tr} \rho$, respectively.

With this notation, we have from Green [1, Theorem 3.8]:

LEMMA 2.4. If $\rho$ is a congruence on $S$, then $\rho=\rho^{\min } \vee \rho_{\min }$.

Throughout the paper we will be interested in various classes of inverse semigroups. In general these will not be varieties but quasivarieties. A quasivariety is a class of algebras defined by a family of implications of the form: $u_{\alpha}=v_{\alpha}(\alpha \in A) \Rightarrow$ $u=v$. For instance, the class of inverse semigroups for which $\mathcal{H}$ is a congruence may be defined as the class of inverse semigroups for which $x x^{-1}=x^{-1} x \Rightarrow x y y^{-1}$ $=y y^{-1} x$.

A congruence $\rho$ on $S$ is idempotent separating if $e^{2}=e, f^{2}=f$ and $e \rho f$ imply that $e=f$. Equivalently, $\rho$ is idempotent separating if and only if $\rho \subseteq \mathcal{K}$. On the other hand $\rho$ is idempotent pure if $\operatorname{ker} \rho=E$; such congruences were called idempotent determined congruences in [1].

Certain specific congruences figure prominently in our discussions. We denote by $\omega, \sigma, \eta, \nu, \pi, \mu$ and $\tau$ the universal, minimum group, minimum semilattice, minimum semilattice of groups, minimum $E$-unitary, maximum idempotent separating and maximum idempotent pure congruences on $S$, respectively.

For any relation $\rho$ on $S$, we will write $\rho^{*}$ for the minimum congruence on $S$ containing $\rho$. We will write

$$
E \zeta=\{a \in S \mid a e=e a, \text { for all } e \in E\}
$$

and denote by $[\rho, \xi]$ the sublattice of $\Lambda(S)$ consisting of those congruences $\theta$ with $\rho \subseteq \theta \subseteq \xi$. We also adopt the convention that, for any $x, y \in S, x y^{0}=x$.

3. Preliminary results on implications in inverse semigroups. The observations of the next lemma will be used frequently.

LEMMA 3.1. For any congruence $\rho$ on $S$, the following statements hold.

(1) $S / \rho$ is an idempotent separating homomorphic image of $S / \rho_{\min }$.

(2) $S / \rho$ is an idempotent pure homomorphic image of $S / \rho^{\mathrm{min}}$.

Proof. (1) The mapping

$$
\varphi: s \rho_{\min } \rightarrow s \rho \quad(s \in S)
$$

is evidently a homomorphism of $S / \rho_{\min }$ onto $S / \rho$. Let $e, f \in E$ and assume that $\left(e \rho_{\min }\right) \varphi=\left(f \rho_{\min }\right) \varphi$. Then $e \rho=f \rho$ so that $e \rho f$. But then $e \rho_{\min } f$ since $\operatorname{tr} \rho=\operatorname{tr} \rho_{\min }$, and thus $e \rho_{\min }=f \rho_{\min }$. Consequently $\varphi$ is idempotent separating.

(2) The mapping

$$
\psi: s \rho^{\min } \rightarrow s \rho \quad(s \in S)
$$


is clearly a homomorphism of $S / \rho^{\min }$ onto $S / \rho$. Let $a \in S$ and $e \in E$ be such that $\left(a \rho^{\mathrm{min}}\right) \psi=\left(e \rho^{\mathrm{min}}\right) \psi$. Then $a \rho=e \rho$ and thus $a \rho e$. It follows that $a \rho^{\min } e$ since $\operatorname{ker} \rho=\operatorname{ker} \rho^{\min }$, and hence $a \rho^{\min }=e \rho^{\min }$. This shows that $\psi$ is idempotent pure.

Underlying much of this paper is the fact that implications can be pulled back under certain kinds of homomorphisms to related implications.

LEMMA 3.2. Let $\varphi$ be a homomorphism of $S$ onto a semigroup $T$.

(1) If $\varphi$ is idempotent separating and T satisfies the implication $\left\{u_{\alpha}=v_{\alpha}\right\}_{\alpha \in A} \Rightarrow w$ $\in E_{T}$, then $S$ satisfies the implication $\left\{u_{\alpha}=v_{\alpha}\right\}_{\alpha \in A} \Rightarrow w \in E \zeta$.

(2) If $\varphi$ is idempotent pure and T satisfies the implication $\left\{u_{\alpha}=v_{\alpha}\right\}_{\alpha \in A} \Rightarrow w \in E_{T} \zeta$, then $S$ satisfies the implication $\left\{u_{\alpha}=v_{\alpha}\right\}_{\alpha \in A}, e \in E \Rightarrow e w \tau$ we.

Proof. (1) Assume that $\left\{u_{\alpha}=v_{\alpha}\right\}_{\alpha \in A}$ is valid in $S$. Then for any substitution of variables in $u_{\alpha}$ and $v_{\alpha}$, we obtain $u_{\alpha}=v_{\alpha}$ and, hence, by applying $\varphi$, we get $u_{\alpha} \varphi=v_{\alpha} \varphi$. Hence we may consider the last equation as a law in $T$. Then $\left\{u_{\alpha} \varphi=v_{\alpha} \varphi\right\}_{\alpha \in A}$ is valid in $T$, and the hypothesis yields $w \varphi \in E_{T}$. For any $e \in E$, we get $(w \varphi)(e \varphi)=(e \varphi)(w \varphi)$ so that $(w e) \varphi=(e w) \varphi$. But then we $\mathcal{H} e w$ since $\varphi$ is idempotent separating and, hence, the congruence it induces is contained in $\mathcal{H}$. It follows that $(w e)(w e)^{-1}=(e w)(e w)^{-1}$, whence $w e w^{-1}=e w w^{-1}$, which gives $w e=$ $e w$. Consequently $w \in E \zeta$ as required.

(2) Assume that $\left\{u_{\alpha}=v_{\alpha}\right\}_{\alpha \in A}$ is valid in $S$. Then $\left\{u_{\alpha} \varphi=v_{\alpha} \varphi\right\}_{\alpha \in A}$ is valid in $T$ and the hypothesis gives $w \varphi \in E_{T} \zeta$. But then $(w e) \varphi=(e w) \varphi$ for all $e \in E$ which implies that we $\tau e w$ for all $e \in E$ since $\varphi$ is idempotent pure so that the congruence induced by it is contained in $\tau$.

Certain implications figure prominently in this paper and we gather some basic observations about them in the following theorem. We denote by $B_{2}$ the Brandt semigroup of rank two with trivial structure group.

THEOREM 3.3. For an inverse semigroup $S$, the following statements are equivalent.

(1) For some positive integer $n>1, y^{n+1}=y^{n} \Rightarrow y^{2}=y$.

(2) For all positive integers $n>1, y^{n+1}=y^{n} \Rightarrow y^{2}=y$.

(3) For some positive integer $n>1, y^{n+1}=y^{n} \Rightarrow y \in E \zeta$.

(4) For all positive integers $n>1, y^{n+1}=y^{n} \Rightarrow y \in E \zeta$.

(5) $S$ does not contain a subsemigroup isomorphic with $B_{2}$.

Proof. Clearly (2) implies (1). Suppose that $y^{n+1}=y^{n} \Rightarrow y^{2}=y$ for some integer $n$. Let $x^{m+1}=x^{m}$, for some $x \in S$ and some positive integer $m$. If $m=n$, then $x^{2}=x$ as required. If $m<n$, then $x^{n+1}=x^{m+1} x^{n-m}=x^{m} x^{n-m}=x^{n}$ and so again $x^{2}=x$. Now assume that $n<m$. Since $x^{m+1}=x^{m}$ it follows that $x^{m}=x^{k}$, for all $k \geqslant m$.

Let $r$ be the least positive integer such that $x^{r}=x^{k}$, for all $k \geqslant r$. Let $p=[r / 2]+1$ where $[r / 2]$ denotes the integral part of $r / 2$. Since $1<n$, it follows that $\left(x^{p}\right)^{n+1}=$ $\left(x^{p}\right)^{n}$. By hypothesis, $\left(x^{p}\right)^{2}=x^{p}$, from which it easily follows that $x^{p}=x^{k}$ for $k \geqslant p$. Since $p<r$ unless $r=1$ or 2 , we must have $p=1$ or 2 . Hence $x^{n+1}=x^{n}$ and, by hypothesis, $x^{2}=x$, as required. Thus (1) implies (2) and they are equivalent. 
Now suppose that, for some positive integer $n, y^{n+1}=y^{n} \Rightarrow y \in E \zeta$. Let $y \in S$ be such that $y^{n+1}=y^{n}$. Since $y \in E \zeta$, it follows that $y$ lies in a group $\mathcal{H}$-class. But in any group $y^{n+1}=y^{n}$ implies $y^{2}=y$. Thus the implications

$$
y^{n+1}=y^{n} \Rightarrow y \in E \zeta \quad \text { and } y^{n+1}=y^{n} \Rightarrow y^{2}=y
$$

are clearly equivalent. Hence it follows that (1)-(4) are equivalent.

We now show that (2) implies (5).

Since $y^{3}=y^{2}$ is a law satisfied by $B_{2}$ and not every element of $B_{2}$ is an idempotent, it follows that if $S$ satisfies (1) with $n=2$, then it cannot contain (an isomorphic copy of) $B_{2}$.

Finally, we show that (5) implies (1), with $n=2$. Suppose that $y \in S$ is such that $y^{3}=y^{2}$ but $y^{2} \neq y$. Let $T=\left\{y, y^{-1}, y y^{-1}, y^{-1} y, y^{2}\right\}$. We show that $T$ is isomorphic to $B_{2}$. First we must show that the five elements are all distinct.

If $y=y^{-1}$ then $y^{2}=y^{3}=y y y=y y^{-1} y=y$, a contradiction.

If $y=y y^{-1}$ then $y^{2}=y y^{-1} y=y$, again a contradiction.

Continuing in this way one can verify that all five elements are distinct. We shall complete the proof if we can show that all products of the form $y y, y^{-1} y^{-1}, y\left(y y^{-1}\right)$, $y^{-1}\left(y^{-1} y\right)$ and all products involving $y^{2}$ (but not products of the form $\left.\left(y y^{-1}\right) y\right)$ are equal to $y^{2}$, so that $y^{2}$ is the zero of $T$.

Since $y^{3}=y^{2}$, we have $y^{4}=y^{3}=y^{2}$ and so $y^{2}$ is an idempotent. Hence $y^{2}=$ $\left(y^{2}\right)^{-1}=y^{-2}$. Also, for example,

$$
y\left(y y^{-1}\right)=y^{2} y^{-1}=y^{3} y^{-1}=y^{2}\left(y y^{-1}\right)=y y^{-1} y^{2}=y^{2} .
$$

All other products of this type can similarly be reduced to $y^{2}$. Hence $T$ is isomorphic to $B_{2}$.

Thus if $S$ does not contain $B_{2}$ and $y \in S$ is such that $y^{3}=y^{2}$ then we must have $y^{2}=y$. Therefore, the first statement is verified and the proof of the theorem is complete.

According to McAlister [5, Lemma 2.9], the semigroups in the above theorem coincide with the $E$-reflexive inverse semigroups of O'Carroll [7].

Notation 3.4. Much of this paper relates to semigroups satisfying the implications of Theorem 3.3. Since they are all equivalent, we will work with the simplest and will denote it by $(\mathrm{P})$ :

$$
y^{3}=y^{2} \Rightarrow y^{2}=y .
$$

If $\rho$ is a congruence on $S$ such that $S / \rho$ satisfies (P), we will call $\rho$ a $P$-congruence. Since such implications define quasivarieties we provide the following consequence of Theorem 3.3.

COROLlary 3.5. Let $C$ be the quasivariety of inverse semigroups defined by the implication (P). Then $S$ belongs to $C$ if and only if $B_{2}$ is not a subsemigroup of $S$.

4. Characterizations of $\gamma_{\min },\left(\gamma_{\min }\right)^{\min }$ and $\left(\left(\gamma_{\min }\right)^{\min }\right)_{\min }$. We will now develop characterizations of the three congruences $\gamma_{\min },\left(\gamma_{\min }\right)^{\min }$ and $\left(\left(\gamma_{\min }\right)^{\min }\right)_{\min }$ for any congruence $\gamma$ on $S$, where for the last two, $\gamma$ is also a $P$-congruence. 
From the definition of $\gamma_{\min }$ as the minimum congruence with the same trace as $\gamma$, the following lemma is a straightforward transcription of Lemma 2.1.

LEMMA 4.1. If $\gamma$ is a congruence on $S$, then

$$
\gamma_{\min }=\left\{(a, b) \mid a^{-1} a \gamma b^{-1} b \text { and } a e=b e, \text { for some e } \gamma a^{-1} a\right\} .
$$

Notation 4.2. In order to simplify the notations we will sometimes write $\gamma_{2}$ for $\left(\gamma_{\min }\right)^{\min }$ and $\gamma_{3}$ for $\left(\left(\gamma_{\min }\right)^{\min }\right)_{\min }$.

THEOREM 4.3. Let $n$ be a positive integer and let $\gamma$ be a P-congruence on $S$. Then $\left(\gamma_{\min }\right)^{\mathrm{min}}$ is the minimum congruence $\rho$ on $S$ such that

$$
x y^{n} \rho x y^{n-1}, x \gamma y \Rightarrow y^{2} \rho y .
$$

Proof. Since $\gamma$ is a $P$-congruence, it satisfies the implication $\left(\mathrm{Q}_{n}\right)$, by Theorem 3.3. Clearly the intersection of any family of congruences satisfying $\left(\mathrm{Q}_{n}\right)$ is again a congruence satisfying $\left(\mathrm{Q}_{n}\right)$ and so there is a minimum such congruence.

Let $\rho$ be any congruence on $S$ such that $\operatorname{ker} \rho=\operatorname{ker} \gamma_{\min }$ and $\rho \subseteq \gamma_{\min }$. Let $x$, $y \in S$ be such that $x y^{n} \rho x y^{n-1}$ and $x \gamma y$. Then $\rho \subseteq \gamma_{\min } \subseteq \gamma$ implies that $y^{n+1} \gamma y^{n}$ which, by (P) and Theorem 3.5, implies that $y^{2} \gamma y$. Since $\gamma / \gamma_{\min }$ is an idempotent separating congruence, it follows that $x \gamma_{\min }$ and $y \gamma_{\min }$ lie in the same group $\mathcal{H}$-class in $S / \gamma_{\min }$. But $x y^{n} \gamma_{\min } x y^{n-1}$ and so $y \gamma_{\min }$ must be the idempotent of that group $\mathcal{H}$-class. Since $\gamma_{\min }$ and $\rho$ have the same kernel, it follows that $y^{2} \rho y$.

Thus every congruence which is contained in $\gamma_{\min }$ and has the same kernel as $\gamma_{\min }$ satisfies $\left(\mathrm{Q}_{n}\right)$. In particular, $\gamma_{2}=\left(\gamma_{\min }\right)^{\mathrm{min}}$ satisfies $\left(\mathrm{Q}_{n}\right)$.

Now let $\rho$ be the minimum congruence satisfying $\left(\mathrm{Q}_{n}\right)$. By the above $\rho \subseteq \gamma_{2}$. Hence $\operatorname{ker} \rho \subseteq \operatorname{ker} \gamma_{2}$ and, by the definition of $\gamma_{2}$ as the minimum element in its kernel class, it suffices to show that $\operatorname{ker} \rho \supseteq \operatorname{ker} \gamma_{2}$.

We have

$$
\begin{aligned}
\operatorname{ker} \gamma_{2} & =\operatorname{ker}\left(\gamma_{\min }\right)^{\min }=\operatorname{ker} \gamma_{\min } \\
& =\left\{a \mid a e=e, \text { for some } e^{2}=e \gamma a^{-1} a\right\} .
\end{aligned}
$$

Let $e^{2}=e \gamma a^{-1} a$ and $a e=e$. Then $e \gamma a$ and $a^{n} e=a^{n-1} e$ while $e \gamma a^{-1} a \gamma a$. Since $\rho$ satisfies $\left(\mathrm{Q}_{n}\right), a^{2} \rho a$. Thus $a \in \operatorname{ker} \rho$, as required, and $\rho=\left(\gamma_{\min }\right)^{\mathrm{min}}$.

In the next two lemmas we provide some further information on congruences satisfying the implications $\left(\mathrm{Q}_{n}\right)$.

LEMMA 4.4. Let $\gamma$ be a P-congruence on $S$. Let $\rho$ be a congruence on $S$ such that $\rho \subseteq \gamma_{\min }$. Then the following statements are equivalent.

(1) There exists a positive integer $n$ such that $\rho$ satisfies $\left(Q_{n}\right)$.

(2) For all integers $n, \rho$ satisfies $\left(Q_{n}\right)$.

(3) $\rho \in\left[\left(\gamma_{\min }\right)^{\min }, \gamma_{\min }\right]$.

Proof. Clearly (2) implies (1). Let $n$ be a positive integer such that $\rho$ satisfies $\left(\mathrm{Q}_{n}\right)$. By Theorem 4.3, $\left(\gamma_{\min }\right)^{\mathrm{min}}$ is the minimum such congruence and so (3) holds. Thus (1) implies (3). Now suppose that (3) holds and let $n$ be any positive integer. 
Let $x, y$ be such that $x y^{n} \rho x y^{n-1}$ and $x \gamma y$. Since $\rho \subseteq \gamma_{\min } \subseteq \gamma, y^{n+1} \gamma y^{n}$. By hypothesis, $y^{2} \gamma y$. Since $\gamma / \gamma_{\min }$ is an idempotent separating congruence and $x \gamma=$ $y \gamma=y^{2} \gamma$, it follows that $x \gamma_{\min }$ and $y \gamma_{\min }$ belong to the same group $\mathcal{H}$-class in $S / \gamma_{\min }$. Since $\rho \subseteq \gamma_{\min }$, we also have $x y^{n} \gamma_{\min } x y^{n-1}$ which implies that $y \gamma_{\min }$ is the identity of the subgroup to which it belongs. Thus $y \in \operatorname{ker} \gamma_{\min }=\operatorname{ker} \rho$. Therefore $y^{2} \rho y$ and $\rho$ satisfies $\left(\mathrm{Q}_{n}\right)$, as required.

Lemma 4.5. Let $\gamma$ be a P-congruence on $S$. Then any congruence $\rho \in\left[\gamma_{\min }, \gamma\right]$ satisfies $\left(\mathrm{Q}_{n}\right)$ for all positive integers $n$.

Proof. Let $x, y \in S$ be such that $x y^{n} \rho x y^{n-1}$ and $x \gamma y$. Since $\rho \subseteq \gamma$, we have that $y^{n+1} \gamma y^{n}$ and so, by hypothesis, $y^{2} \gamma y$. Now $\gamma / \gamma_{\min }$ is an idempotent separating congruence and thus so also is $\gamma / \rho$. Therefore $x \rho=y \rho=(y \rho)^{2}$ must lie in a group $\mathcal{H}$-class of $S / \rho$. But this, combined with $x y^{n} \rho x y^{n-1}$, clearly implies that $y^{2} \rho y$. Hence $\rho$ satisfies $\left(\mathrm{Q}_{n}\right)$.

Notation 4.6. The implications $\left(\mathrm{Q}_{n}\right)$, which figure in the characterization of $\left(\gamma_{\min }\right)^{\mathrm{min}}$ in Theorem 4.3, were shown to be equivalent in Lemma 4.4. In view of this, we will henceforth work with the simplest one, viz. with $\left(\mathrm{Q}_{1}\right)$, and denote it as $(\mathrm{Q})$ :

$$
x y \rho x, x \gamma y \Rightarrow y^{2} \rho y .
$$

It will be shown in Lemma 4.13 that if $\gamma$ is a $P$-congruence and $\rho$ satisfies $(\mathrm{Q})$, then $\rho$ also is a $P$-congruence.

The next result provides a characterization of $\left(\left(\gamma_{\min }\right)^{\min }\right)_{\min }$.

TheOREM 4.7. Let $\gamma$ be a P-congruence on $S$. Let $n$ be a positive integer. Then $\gamma_{3}=\left(\left(\gamma_{\min }\right)^{\min }\right)_{\min }$ is the minimum congruence $\rho$ such that

$$
x y^{n} \rho x y^{n-1}, x \gamma y, e \in E \Rightarrow \text { ey } \rho \text { ye. }
$$

Proof. By Theorem 3.3, $\gamma$ satisfies $\left(\mathrm{R}_{n}\right)$ and it is clear that the intersection of all the congruences satisfying $\left(\mathrm{R}_{n}\right)$ is another such congruence. Hence there is a minimum such congruence, which we denote by $\theta$. Our task is to show that $\theta=\gamma_{3}$.

First let $\rho$ be any congruence such that $\rho \subseteq \gamma_{2}$ and with the same trace as $\gamma_{2}$. Let $x, y$ be such that $x y^{n} \rho x y^{n-1}$ and $x \gamma y$. Then $x y^{n} \gamma_{2} x y^{n-1}$ and $x \gamma y$. By Theorem 4.3 we have $y^{2} \gamma_{2} y$. Since $\gamma_{2} / \rho$ is an idempotent separating congruence on $S / \rho$, it follows that $y \rho$ must be contained in the centralizer of the idempotents of $S / \rho$. Therefore $\rho$ satisfies $\left(R_{n}\right)$. Since $\gamma_{3}$ satisfies the hypothesis on $\rho$, we have that $\gamma_{3}$ satisfies $\left(\mathrm{R}_{n}\right)$. Hence $\theta \subseteq \gamma_{3}$.

In order to establish the converse inclusion, it will suffice to show that $\theta$ and $\gamma_{3}$ have the same trace, since the definition of $\gamma_{3}$ will then imply that $\gamma_{3} \subseteq \theta$. We will accomplish this by showing that there exists a congruence $\rho$ on $S$ such that

(a) $\theta \subseteq \rho$;

(b) $\theta$ and $\rho$ have the same trace;

(c) $\rho$ satisfies $(\mathrm{Q})$. 
For such a congruence $\rho$ we will have

$$
\begin{aligned}
\operatorname{tr} \gamma_{2} & =\operatorname{tr} \gamma_{3} \supseteq \operatorname{tr} \theta, \quad \text { since } \theta \subseteq \gamma_{3}, \\
& =\operatorname{tr} \rho, \quad \text { by }(\mathrm{b}), \\
& \supseteq \operatorname{tr} \gamma_{2}, \quad \text { by Theorem } 4.3 .
\end{aligned}
$$

Hence, $\operatorname{tr} \rho=\operatorname{tr} \gamma_{2}=\operatorname{tr} \gamma_{3}$, as required.

We will establish the existence of $\rho$ by means of a sequence of lemmas.

Lemma 4.8. Let $T=S / \theta$ and $\delta=\gamma / \theta$. Then $\delta$ is a P-congruence and

$$
x y^{n}=x y^{n-1}, x \delta y, e \in E_{T} \Rightarrow e y=y e .
$$

Proof. Let $y=b \theta$. Then $y^{3} \delta y^{2}$ implies that $(b \theta)^{3} \delta(b \theta)^{2}$ and so $b^{3} \gamma b^{2}$. By our assumption about $\gamma$, it follows that $b^{2} \gamma b$ and so $y^{2}=(b \theta)^{2} \delta y=b \theta$. Therefore $\delta$ is a $P$-congruence on $T$.

To see that $(\mathrm{K})$ is also satisfied, let $x=a \theta$ and suppose that $x y^{n}=x y^{n-1}$ and $x \delta y$. Then $a b^{n} \theta a b^{n-1}$ and $a \gamma b$. By the definition of $\theta$, it follows that $e b \theta b e$, for all $e \in E$. Hence $z y=y z$, for all $z \in E_{T}$.

Since the notation would be extremely cumbersome if we were to construct the desired congruence $\rho$ on $S$ directly we will, in fact, construct a congruence on $T$, which will be easier to describe since it will be idempotent separating on $T$, and will then pull it back to $S$.

In the next lemma, we show that if the congruence that we construct on $T$ has suitable properties, then it will lift to $S$ to give us a suitable congruence on $S$.

LEMMA 4.9. Let $\tau$ be an idempotent separating congruence on $T$ such that

$$
x y^{n} \tau x y^{n-1}, x \delta y \Rightarrow y^{2} \tau y .
$$

Let $\rho$ be the congruence on $S$ such that $\tau=\rho / \theta$. Then $\rho$ satisfies (a), (b) and (c) in the first part of the proof of Theorem 4.7 .

Proof. From the definition of $\rho$, we must have $\theta \subseteq \rho$ and so (a) is satisfied. Since $\tau=\rho / \theta$ is idempotent separating, (b) is satisfied. Finally, let $x, y \in S$ be such that $x y^{n} \rho x y^{n-1}$ and $x \gamma y$. Then $(x \theta)(y \theta)^{n} \tau(x \theta)(y \theta)^{n-1}$ and $(x \theta) \delta(y \theta)$. By (L) we have $(y \theta)^{2} \tau(y \theta)$ and so $y^{2} \rho y$. Thus (c) is satisfied and the proof is complete.

In order to complete the proof of Theorem 4.7 , it therefore only remains to establish the following lemma.

LEMMA 4.10. There exists an idempotent separating congruence $\tau$ on $T$ such that $(\mathrm{L})$ holds.

Proof. For each $e \in E=E_{T}$, let

$$
N_{e}=\left\{a \in H_{e} \mid f a=f, \text { for some } f \delta e\right\}
$$

and let $N=\cup\left\{N_{e} \mid e \in E\right\}$. We wish to show that this is the kernel of an idempotent separating congruence on $T$. By Scheiblich [11], it suffices to show that $N$ satisfies the following three conditions:

(1) $E \subseteq N \subseteq E \zeta$; 
(2) $N$ is an inverse subsemigroup of $T$;

(3) $N$ is self-conjugate, that is, $x^{-1} N x \subseteq N$, for all $x \in S$.

That $E \subseteq N$ is clear. Also, $a \in N_{e}$ implies that $f a=f$, for some $f^{2}=f \delta e$. Hence ( $f a) a^{n}=(f a) a^{n-1}$, where $f a \delta a a^{-1} a=a$. By Lemma 4.8, this implies that $a \in E \zeta$ and so (1) holds.

Now $a \in N_{e}, b \in N_{f}$ implies that $g a=g$ and $h b=h$ for some $g^{2}=g \delta a a^{-1}$, $h^{2}=h \delta b b^{-1}$. Then

$$
\left(a^{-1} g a\right) a^{-1}=a^{-1} g=(g a)^{-1}=g
$$

where $a^{-1} g a \delta a^{-1} a a^{-1} a=a^{-1} a$. Thus $a^{-1} \in N_{e}$. Also, since $N \subseteq E \zeta$,

$$
g h a b=(g a)(h b)=g h
$$

where $g h \delta a a^{-1} b b^{-1}=a b b^{-1} a^{-1}=(a b)(a b)^{-1}$. Since

$$
(a b)(a b)^{-1}=a b b^{-1} a^{-1}=a a^{-1} b b^{-1}
$$

and

$$
(a b)^{-1}(a b)=b^{-1} a^{-1} a b=a^{-1} a b^{-1} b=a a^{-1} b b^{-1},
$$

we have $a b \in H_{e}$, where $e=a a^{-1} b b^{-1}$, and so $a b \in N_{e}$. Thus (2) holds.

Next, let $a$ and $g$ be as above and $x \in S$. Then $\left(x^{-1} g x\right)\left(x^{-1} a x\right)=x^{-1} g a x=x^{-1} g x$ where

$$
x^{-1} g x \delta x^{-1} a a^{-1} x=x^{-1} a x x^{-1} a^{-1} x=\left(x^{-1} a x\right)\left(x^{-1} a x\right)^{-1} .
$$

Therefore (3) holds and $N$ is the kernel of an idempotent separating congruence $\tau$, say.

For the next part, we need to know that $\tau \subseteq \delta$. Let $x \in \operatorname{ker} \tau=N$, say $x \in N_{e}$ where $e=x x^{-1}$. Then for some $f \delta e, f x=f$ and so

$$
x=x x^{-1} x \delta f x=f .
$$

Thus $x \in \operatorname{ker} \delta$ and $\operatorname{ker} \tau \subseteq \operatorname{ker} \delta$. Since $\tau$ is idempotent separating, it follows that $\tau \subseteq \delta$.

Finally, we show that $\tau$ satisfies (L). Let $x$ and $y$ be such that $x y^{n} \tau x y^{n-1}$ and $x \delta y$. Since $\tau \subseteq \delta$, it follows from this that $y^{n+1} \delta y^{n}$ and, since $\delta$ is a $P$-congruence, $y^{2} \delta y$. Hence $x, y, x^{-1}, y^{-1}$ and $y y^{-1}$ are all $\delta$-equivalent. On the other hand, $x y^{n} \tau x y^{n-1}$ implies, since $\tau$ is idempotent separating, that $x y^{n}=h x y^{n-1}$ for some $h \in N_{e}$, where $e=\left(x y^{n}\right)\left(x y^{n}\right)^{-1}$. Then there must exist an idempotent $f$ such that $f \delta e$ and $f h=f$. For this $f$, it follows that $f x y^{n}=f h x y^{n-1}=f x y^{n-1}$, where

$$
f x \delta e x=\left(x y^{n}\right)\left(x y^{n}\right)^{-1} x \delta y
$$

since $x, y$ and $y y^{-1}$ are all $\delta$-equivalent. Therefore, by (K), $y \in E \zeta$ and so $y \in H_{g}$, for some $g \in E$.

Furthermore, it follows from $f x y^{n}=f x y^{n-1}$ that

$$
\left(y^{n-1} x^{-1} f x y^{n-1}\right) y=y^{n-1} x^{-1} f x y^{n-1}
$$

where, again since $x, y$ and $y y^{-1}$ are $\delta$-equivalent,

$$
y^{n-1} x^{-1} f x y^{n-1} \delta y y^{-1} .
$$


Hence $y \in N_{g}$ and so $y^{2} \tau y$, as required. Therefore, $\tau$ satisfies (L), the proof of the lemma is complete and also the verification of Theorem 4.7.

The next result relating to $\gamma_{3}$ and $\left(R_{n}\right)$ is parallel to Lemma 4.4 on $\left(\gamma_{\min }\right)^{\min }$ and $\left(\mathrm{Q}_{n}\right)$.

LeMma 4.11. Let $\gamma$ be a P-congruence on $S$. Let $\rho$ be a congruence on $S$ such that $\rho \subseteq \gamma_{\min }$. Then the following statements are equivalent.

(1) There exists a positive integer $n$ such that $\rho$ satisfies $\left(R_{n}\right)$.

(2) For all positive integers $n$, $\rho$ satisfies $\left(\mathrm{R}_{n}\right)$.

(3) $\rho \in\left[\gamma_{3}, \gamma_{\min }\right]$.

Proof. Clearly (2) implies (1) and, by the minimality of $\gamma_{3}$, (1) implies (3). Now let (3) hold and let $n$ be a positive integer. Let $x, y \in S$ be such that $x y^{n} \rho x y^{n-1}$ and $x \gamma y$. Since $\rho \subseteq \gamma_{\min } \subseteq \gamma, y^{n+1} \gamma y^{n}$. By hypothesis $y^{2} \gamma y$. Since $\gamma / \gamma_{\min }$ is an idempotent separating congruence and $x \gamma=y \gamma=y^{2} \gamma$ it follows, as in Lemma 4.4, that $y^{2} \gamma_{\min } y$. Hence $y^{2} \gamma_{2} y$ and so ey $\gamma_{3} y e$, for all $e \in E$. Since $\gamma_{3} \subseteq \rho$, we have that ey $\rho$ ye, for all $e \in E$. Thus $\rho$ satisfies $\left(\mathrm{R}_{n}\right)$, as required.

Notation 4.12. In the light of Lemma 4.11, for any congruence $\rho \subseteq \gamma_{\min }$, we may work with the simplest implication, viz. with $\left(\mathrm{R}_{1}\right)$, and denote it by $(\mathrm{R})$ :

$$
x y \rho x, x \gamma y, e \in E \Rightarrow \text { ey } \rho \text { ye. }
$$

Lemma 4.13. Let $\gamma$ be a P-congruence on $S$ and let $\rho$ be a congruence on $S$ satisfying either $(\mathrm{Q})$ or $(\mathrm{R})$. Then $\rho$ is a P-congruence. This holds, in particular, for $\rho=\left(\gamma_{\mathrm{min}}\right)^{\mathrm{min}}$ or $\rho=\left(\left(\gamma_{\min }\right)^{\min }\right)_{\min }$.

PRoof. Since (Q) and (R) have the same hypotheses but (R) has the weaker conclusion, it will suffice to establish the result under the assumption that $\rho$ satisfies (R). Let $y \in S$ be such that $y^{3} \rho y^{2}$. By (R), this implies that ey $\rho$ ye, for all $e \in E$. Therefore $y \rho \in E_{S / \rho} \zeta$ and so, in particular, $y \rho$ lies in a group $\mathcal{H}$-class of $S / \rho$. But $(y \rho)^{3}=(y \rho)^{2}$ and therefore $y^{2} \rho y$ and $\rho$ is a $P$-congruence.

\section{The min network.}

Definition 5.1. On $S$ we define inductively the following two sequences of congruences:

$$
\begin{aligned}
& \alpha_{0}=\omega=\beta_{0}, \\
& \alpha_{n}=\left(\beta_{n-1}\right)_{\min }, \quad \beta_{n}=\left(\alpha_{n-1}\right)^{\min } \text { for } n \geqslant 1 .
\end{aligned}
$$

We call the aggregate $\left\{\alpha_{n}, \beta_{n}\right\}_{n=0}^{\infty}$, together with the inclusion relation for congruences, the min network of congruences on $S$.

We shall see presently that the min network is related to the following family of implications.

Definition 5.2. An inverse semigroup $S$ might satisfy one of the following implications:

$$
\begin{aligned}
& \left(\mathrm{A}_{0}\right): x=y ;\left(\mathrm{A}_{1}\right): x^{-1} x=y^{-1} y ;\left(\mathrm{A}_{2}\right): y \in E \zeta \\
& \left(\mathrm{A}_{n}\right): x y=x, x \beta_{n-3} y \Rightarrow y \in E \zeta, n \geqslant 3 \\
& \left(\mathrm{~B}_{0}\right): x=y ;\left(\mathrm{B}_{1}\right): y \in E
\end{aligned}
$$


$\left(\mathrm{B}_{n}\right): x y=x, x \beta_{n-2} y \Rightarrow y \in E, n \geqslant 2$.

The next few results develop some basic facts about the min network.

Proposition 5.3. For $n \geqslant 1$, we have $\alpha_{n-1} \cap \beta_{n-1}=\alpha_{n} \vee \beta_{n}$.

Proof. Let $\rho=\alpha_{n-1} \cap \beta_{n-1}$; then

$$
\begin{aligned}
\operatorname{tr} \rho & =\operatorname{tr}\left(\alpha_{n-1} \cap \beta_{n-1}\right)=\operatorname{tr} \alpha_{n-1} \cap \operatorname{tr} \beta_{n-1} \\
& =\operatorname{tr} \alpha_{n-1} \cap \operatorname{tr} \alpha_{n}=\operatorname{tr} \alpha_{n}, \\
\operatorname{ker} \rho= & \operatorname{ker}\left(\alpha_{n-1} \cap \beta_{n-1}\right)=\operatorname{ker} \alpha_{n-1} \cap \operatorname{ker} \beta_{n-1} \\
= & \operatorname{ker} \beta_{n} \cap \operatorname{ker} \beta_{n-1}=\operatorname{ker} \beta_{n},
\end{aligned}
$$

which implies $\rho_{\min }=\alpha_{n}$ and $\rho^{\min }=\beta_{n}$. Hence, $\rho=\rho_{\min } \vee \rho^{\min }$ by Lemma 2.4 yields $\alpha_{n-1} \cap \beta_{n-1}=\alpha_{n} \vee \beta_{n}$.

COROLlaRY 5.4. The min network, together with the intersections of corresponding pairs, is a sublattice of $\Lambda(S)$.

The first few levels of the min network are depicted in Diagram 1 together with some relationships and alternative characterizations.

Recall that $S$ is said to be $E$-unitary if $e y=e$ for some $e \in E$ implies that $y \in E$. Equivalently $S$ is $E$-unitary if and only if it satisfies the implication $x y=x \Rightarrow y^{2}=y$. We now come to the main theorem.

THEOREM 5.5. (1) $\alpha_{n}$ is the minimum congruence $\rho$ on $S$ such that $S / \rho$ satisfies $\left(\mathrm{A}_{n}\right)$; moreover, $\alpha_{n}$ is a P-congruence.

(2) $\beta_{n}$ is the minimum congruence $\rho$ on $S$ such that $S / \rho$ satisfies $\left(\mathrm{B}_{n}\right)$; moreover, $\beta_{n}$ is a P-congruence.

Proof. We will first observe that the theorem is true for $n=0,1$ and 2 and then complete the proof with an induction argument.

For $n=0$, we have $\alpha_{0}=\beta_{0}=\omega$, while $A_{0}$ and $B_{0}$ both require that the factor semigroup be trivial. Hence the result is trivial in this case.

The assertion of the theorem for $\alpha_{1}$ follows directly from the fact that congruences with universal traces coincide with group congruences. In particular, $\alpha_{1}=\left(\beta_{0}\right)_{\min }=$ $\omega_{\min }=\sigma$, the least group congruence.

The claim of the theorem for $\beta_{1}$ follows from the fact that congruences with kernels equal to $S$ coincide with semilattice congruences. In particular, $\beta_{1}=\left(\alpha_{0}\right)^{\text {min }}$ $=\omega^{\min }=\eta$, the least semilattice congruence.

For the case $n=2$, we have $\alpha_{2}$ equal to the minimum congruence with the same trace as $\beta_{1}=\eta$, the minimum semilattice congruence. Clearly $\alpha_{2}$ must be a semilattice of groups congruence and so contains $\nu$, the minimum semilattice of groups congruence. But $\nu$ has the same trace as $\eta$ and so $\alpha_{2} \subseteq \nu$. Therefore $\alpha_{2}=\nu$ and so is the minimum congruence on $S$ such that the factor semigroup satisfies $\left(\mathrm{A}_{2}\right)$.

For the last special case, we have that $\beta_{2}$ is the minimum congruence with the same kernel as $\sigma$. It is routine to verify that $\beta_{2}$ must, therefore, be an $E$-unitary congruence. Hence $\beta_{2} \supseteq \pi$, the minimum $E$-unitary congruence. However, it is easy to see that $\operatorname{ker} \pi=\operatorname{ker} \sigma$, and so by the definition of $\beta_{2}$ we must have $\pi \subseteq \beta_{2}$. Thus 


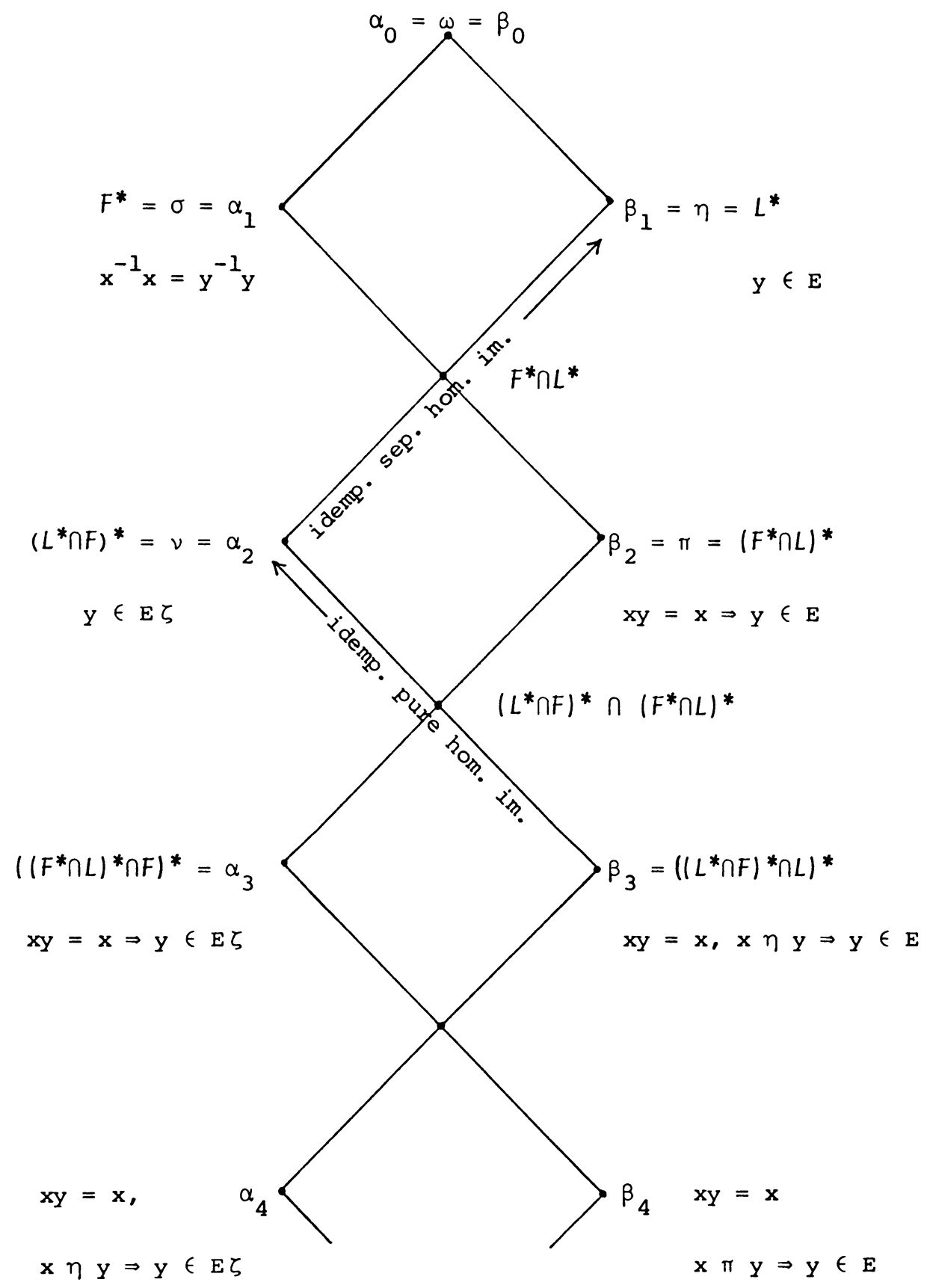

Diagram 1 
$\beta_{2}=\pi$, the minimum congruence $\rho$ such that $S / \rho$ satisfies $\left(\mathrm{B}_{2}\right)$ (see the remark preceding the theorem).

It is a simple matter to verify that each of the specific congruences $\rho=\omega, \sigma, \eta, \nu$ or $\pi$ is a $P$-congruence.

Now suppose that $n \geqslant 3$ and that the theorem is valid for smaller integers. Then, by the induction hypothesis, $\beta_{n-2}$ is a $P$-congruence. Hence we can apply Theorem 4.3 with $\gamma=\beta_{n-2}$ and deduce that $\beta_{n}$ is the minimum congruence $\rho$ on $S$ such that $S / \rho$ satisfies $\left(\mathrm{B}_{n}\right)$. It follows directly from $\left(\mathrm{B}_{n}\right)$ or from Lemma 4.13 that $\beta_{n}$ is a $P$-congruence.

Similarly, applying Theorem 4.7, with $\gamma=\beta_{n-3}$ we obtain that $S / \alpha_{n}$ satisfies $\left(\mathrm{A}_{n}\right)$ and from Lemma 4.13 that $\alpha_{n}$ is a $P$-congruence.

Corollary 5.6. Let $\rho=\bigcap_{n=0}^{\infty} \alpha_{n}$. Then $\rho=\bigcap_{n=0}^{\infty} \beta_{n}$ and $\rho$ is a P-congruence.

Proof. The equality $\rho=\bigcap_{n=0}^{\infty} \beta_{n}$ follows from Proposition 5.3. From Theorem 5.5, $\alpha_{n}$ is a $P$-congruence and it follows readily that $\rho$ also is a $P$-congruence.

\section{Further properties of the min network.}

Proposition 6.1. The following conditions are equivalent for $n>2$.

(1) $S$ is a subdirect product of a semigroup $U$ satisfying $\left(\mathrm{A}_{n-1}\right)$ and a semigroup $V$ satisfying $\left(\mathrm{B}_{n-1}\right)$.

(2) $S$ satisfies $\left(\mathrm{A}_{n}\right)$ and $\left(\mathrm{B}_{n}\right)$.

(3) $S$ satisfies the implication

$$
\left.\begin{array}{ll}
u z=u, & u \beta_{n-3} z \\
x y=x, & x \beta_{n-2} y
\end{array}\right\} \Rightarrow z y^{2}=y z
$$

where for $n=2$ the first part of the hypothesis is omitted.

Proof. (1) implies (2). Let $\alpha$ and $\beta$ be the congruences on $S$ induced by the subdirect product in the hypothesis. Then $S / \alpha$ satisfies $\left(\mathrm{A}_{n-1}\right)$ and $S / \beta$ satisfies $\left(\mathrm{B}_{n-1}\right)$ and $\alpha \cap \beta=\varepsilon$. By the ninimality of $\alpha_{n-1}$ and $\beta_{n-1}$ established in Theorem 5.5 , we conclude that $\alpha_{n-1} \subseteq \alpha$ and $\beta_{n-1} \subseteq \beta$. Hence $\alpha_{n-1} \cap \beta_{n-1}=\varepsilon$, which by Proposition 5.3 yields $\alpha_{n} \vee \beta_{n}=\varepsilon$. But then $\alpha_{n}=\beta_{n}=\varepsilon$ and, hence, by Theorem 5.5, $S$ satisfies both $\left(\mathrm{A}_{n}\right)$ and $\left(\mathrm{B}_{n}\right)$.

(2) implies (1). Theorem 5.5 gives that $\alpha_{n}=\beta_{n}=\varepsilon$ by the minimality of these congruences. By Proposition 5.3, we get $\varepsilon=\alpha_{n} \vee \beta_{n}=\alpha_{n-1} \cap \beta_{n-1}$ and thus $S$ is a subdirect product of $S / \alpha_{n-1}$ and $S / \beta_{n-1}$, where the first satisfies $\left(\mathrm{A}_{n-1}\right)$ and the second $\left(\mathrm{B}_{n-1}\right)$, by Theorem 5.5 .

(2) implies (3). Assume $u z=u, u \beta_{n-3} z, x y=x, x \beta_{n-2} y$, with the convention for $n=2$. From $\left(\mathrm{A}_{n}\right)$ we get $z \in E \zeta$ and from $\left(\mathrm{B}_{n}\right)$ we have $y \in E$ so that $z y^{2}=y z$.

(3) implies (1). Assume $x y=x, x \beta_{n-3} y$ with the convention for $n=2$. Then for $e \in E$, we have $e e=e$ and $e \beta_{n-2} e$ so that the hypothesis yields $e y^{2}=y e$. In particular, for $e=y^{-1} y$ and $e=y y^{-1}$, we obtain $y=y^{-1} y^{3}$ and $y^{2}=y^{2} y^{-1}$, whence

$$
\begin{aligned}
y & =y^{-1} y^{2} y=y^{-1}\left(y^{2} y^{-1}\right) y=y^{-1} y^{2}=y^{-1}\left(y^{2} y^{-1}\right) \\
& =\left(y^{-1} y\right)\left(y y^{-1}\right) \in E .
\end{aligned}
$$


Green [1] introduced the following relation on any inverse semigroup $S$ :

$$
a \mathscr{F} b \Leftrightarrow a b^{-1} \in E_{S} .
$$

The next theorem exhibits several relationships among congruences on $S$, the relation $\mathscr{F}$ and Green's relation $\mathscr{L}$, and has a number of interesting consequences.

THEOREM 6.2. The following statements concerning congruences $\rho$ and $\xi$ on $S$ are valid.

(1) If $\operatorname{tr} \rho \subseteq \operatorname{tr} \xi$, then

$$
\begin{gathered}
\rho \vee \xi_{\min }=\xi_{\min } \rho \xi_{\min }, \\
a \rho \vee \xi_{\min } b \Leftrightarrow a e \rho b e, \quad e \xi a^{-1} a \xi b^{-1} b \quad \text { for some } e \in E .
\end{gathered}
$$

(2) If $\rho \subseteq \xi$, then

$$
\begin{aligned}
& \rho \vee \xi_{\min }=(\xi \cap \rho \mathscr{F})^{*}, \\
& \rho \vee \xi^{\min }=(\xi \cap \rho \mathcal{L})^{*} .
\end{aligned}
$$

Proof. (1) Assume that $\operatorname{tr} \rho \subseteq \operatorname{tr} \xi$. The inclusion $\xi_{\min } \rho \xi_{\min } \subseteq \rho \vee \xi_{\min }$ is obvious. For the opposite inclusion, it suffices to show that $\rho \xi_{\min } \rho \subseteq \xi_{\min } \rho \xi_{\min }$. Hence let $a, b, c, d \in S$ be such that $a \rho b \xi_{\min } c \rho d$. Then $a^{-1} a \rho b^{-1} b$ and thus $a^{-1} a \xi b^{-1} b$. Likewise $c^{-1} c \xi d^{-1} d$. Also, $b e=c e$ and $e \xi b^{-1} b \xi c^{-1} c$ for some $e \in E$. Thus $e \xi a^{-1} a \xi d^{-1} d$ and

$$
a e \rho b e=c e \rho d e,
$$

which implies that $a \xi_{\min } a e \rho d e \xi_{\min } d$. Consequently $\rho \xi_{\min } \rho \subseteq \xi_{\min } \rho \xi_{\min }$, and the assertion follows.

Next let $a \rho \vee \xi_{\min } b$. Then (A) yields $a \xi_{\min } c \rho d \xi_{\min } b$ for some $c, d \in S$. Hence for some $e \in E$, we have $a e=c e$ and $e \xi a^{-1} a \xi c^{-1} c$. Likewise, for some $f \in E$, we have $d f=b f$ and $f \xi d^{-1} d \xi b^{-1} b$. Now $c^{-1} c \rho d^{-1} d$ and so, by hypothesis, $c^{-1} c \xi d^{-1} d$. Therefore $e \xi f$ and we have

$$
a e f=c e f \rho \operatorname{def}=b e f
$$

where ef $\xi a^{-1} a \xi b^{-1} b$.

Conversely, if $a e \rho b e$ and $e \xi a^{-1} a \xi b^{-1} b$ for some $e \in E$, then

$$
a \xi_{\min } a e \rho b e \xi_{\min } b \text {, }
$$

which by (A) gives $a \rho \vee \xi_{\min } b$.

(2) Assume that $\rho \subseteq \xi$. Since $\mathscr{F}$ contains the diagonal, we have $\rho \subseteq \xi \cap \rho^{\mathscr{F}}$ and thus $\rho \subseteq\left(\xi \cap \rho^{\mathscr{F}}\right)^{*}$. Finally $\operatorname{tr} \xi \subseteq \operatorname{tr}(\xi \cap \rho \mathscr{F})^{*}$ and so $\xi_{\min } \subseteq\left(\xi \cap \rho_{\mathscr{F}}\right)^{*}$. Consequently $\rho \vee \xi_{\min } \subseteq(\xi \cap \rho \widetilde{F})^{*}$.

For the converse, let $a \xi \cap \rho \mathscr{F} b$. Then $a^{-1} a \xi b^{-1} b$ and so $a^{-1} a \rho \vee \xi_{\min } b^{-1} b$. Also $a \rho x \mathscr{F} b$, for some $x \in S$. Hence $a b^{-1} \rho x b^{-1} \in E$, by the definition of $\mathscr{F}$. Thus $a b^{-1} \rho \vee \xi_{\min } e$ where $e=x b^{-1} \in E$. In the light of Lemma 2.2, we conclude that $a \rho \vee \xi_{\min } b$. It follows that $\left(\xi \cap \rho^{\mathscr{F}}\right)^{*} \subseteq \rho \vee \xi_{\text {min }}$.

The hypothesis implies that $\rho \subseteq \xi \cap \rho \mathscr{L}$ and thus $\rho \subseteq(\xi \cap \rho \mathscr{L})^{*}$. Let $a \xi e$ where $e \in E$. Then $a \xi a^{-1} a$ which, together with $a \varrho a^{-1} a$, yields $a \xi \cap \rho \varrho a^{-1} a$. It follows that $a(\xi \cap \rho \mathscr{L})^{*} a^{-1} a$, which implies that $\operatorname{ker} \xi \subseteq \operatorname{ker}(\xi \cap \rho \mathscr{L})^{*}$. By the minimality of $\xi^{\min }$ we conclude that $\xi^{\min } \subseteq(\xi \cap \rho \mathscr{L})^{*}$. 
For the opposite inclusion let $\kappa$ be a congruence on $S$ such that $\rho \subseteq \kappa$ and $\xi^{\text {min }} \subseteq \kappa$. Letting $\theta=\kappa \cap \xi$, we obtain $\rho \subseteq \theta$ and

$$
\operatorname{ker} \xi=\operatorname{ker} \xi^{\min } \subseteq \operatorname{ker}(\kappa \cap \xi)=\operatorname{ker} \kappa \cap \operatorname{ker} \xi \subseteq \operatorname{ker} \xi
$$

so that $\operatorname{ker} \xi=\operatorname{ker} \theta$. Now let $a \xi \cap \rho \mathcal{L}$. Then $a \xi b$ and $a \rho x, x^{-1} x=b^{-1} b$ for some $x \in S$. Hence $a b^{-1} \xi b b^{-1}$ which gives $a b^{-1} \in \operatorname{ker} \xi=\operatorname{ker} \theta$, and $a^{-1} a \rho b^{-1} b$ which implies $a^{-1} a \theta b^{-1} b$. In view of Lemma 2.2, we conclude that $a \theta b$. But then also $a \kappa b$, which shows that $\xi \cap \rho \mathcal{L} \subseteq \kappa$. Consequently, $(\xi \cap \rho \mathcal{L})^{*} \subseteq \kappa$ and, in particular, $(\xi \cap \rho \mathcal{L})^{*} \subseteq \rho \vee \xi^{\min }$.

REMARKS 6.3. The results in Theorem 6.2 reduce to a number of interesting special cases as follows:

Item (A) in the case $\xi=\omega$ gives $\rho \vee \sigma=\sigma \rho \sigma$, which is due to Howie [3, Theorem 3.9].

Item (B) in the case $\rho=\varepsilon$ yields

$$
a \xi_{\min } b \Leftrightarrow a e=b e, \quad e \xi a^{-1} a \xi b^{-1} b,
$$

which was established by Reilly and Scheiblich [10, Theorem 4.2]; thus (B) can be viewed as its generalization.

Consider now item (C). For $\xi=\omega$ we get the formula $\rho \vee \sigma=(\rho \mathscr{F})^{*}$ and for $\rho=\varepsilon$, the formula $\xi_{\min }=(\xi \cap \mathscr{F})^{*}$. The conjunction of these two conditions gives $\sigma=\mathscr{F}^{*}$, proved by Green [1, Proposition 2.3].

Finally consider item (D). For $\xi=\omega$, we get $\rho \vee \eta=(\rho \varrho)^{*}$ and for $\rho=\varepsilon$, we have $\xi^{\mathrm{min}}=(\xi \cap \mathcal{L})^{*}$. The conjunction of these two conditions yields $\eta=\mathcal{L}^{*}$ which is due to Howie and Lallement [4, Theorem 1.6]. In the case $\rho=\varepsilon$ and $\xi=\sigma$, we obtain $\sigma^{\min }=(\sigma \cap \mathcal{L})^{*}$, according to Theorem $4.3, \sigma^{\min }=\left(\omega_{\min }\right)^{\min }$ is the least $E$-unitary congruence on $S$. Hence $(\sigma \cap \mathcal{L})^{*}$ is the least $E$-unitary congruence, a result proved by O'Carroll [6, Theorem 2]. For the case $\rho=\varepsilon$ and $\xi=\nu$, where $\nu$ is the least semilattice of groups congruence, we get by (D), that $\nu^{\min }=(\nu \cap \mathcal{L})^{*}$. According to O'Carroll [7, Proposition 2], $(\nu \cap \mathcal{L})^{*}$ is the least strongly $E$-reflexive congruence. Since $\nu=\alpha_{2}$, we obtain by Theorem 5.5 that $\nu^{\text {min }}=\beta_{3}$ is the least congruence which is a semilattice of $E$-unitary semigroups. Thus we deduce that strongly $E$-reflexive inverse semigroups coincide with semigroups which are semilattices of $E$-unitary inverse semigroups, a result due to O'Carroll [7, Theorem 1].

A further consequence of the theorem follows.

COROLlaRY 6.4. Each of the classes defined by the implications $\left(\mathrm{A}_{n}\right)$ and $\left(\mathrm{B}_{n}\right)$ is a quasivariety.

Proof. According to Theorem 6.2, $\rho_{\min }=(\rho \cap \mathcal{F})^{*}$, so the relation $a \rho_{\min } b$ can be expressed in terms of a finite number of equations and the relation $\rho$. The same type of statement is valid for $\rho^{\min }$. The classes defined by $\left(A_{0}\right),\left(A_{1}\right),\left(B_{0}\right),\left(B_{1}\right)$ are even varieties. Hence by Theorem 5.5, an inductive argument can be used to prove that any $\left(\mathrm{A}_{n}\right)$ and $\left(\mathrm{B}_{n}\right)$ can be given by a system of implications, since for $n \geqslant 3$, $\left(\mathrm{A}_{n}\right)$ and $\left(\mathrm{B}_{n}\right)$ are defined by means of $\beta_{n-3}$ and $\beta_{n-2}$, and these can be obtained from $\alpha_{n-4}$ and $\alpha_{n-3}$ by the procedure mentioned above. 
It would, however, be desirable to have a simple inductive formula for implications defining $\left(\mathrm{A}_{n}\right)$ and $\left(\mathrm{B}_{n}\right)$. For example, we have

$\mathrm{B}_{0}: x=y$ (trivial semigroups),

$\mathrm{B}_{1}: y^{2}=y$ (semilattices),

$\mathrm{B}_{2}: x y^{2}=x y \Rightarrow y^{2}=y(E$-unitary $)$,

$\mathrm{B}_{3}:(x y)^{2}=x y \Rightarrow ;(y x)^{2}=y x, e=e^{2},(e x y)^{2}=e x y \Rightarrow(\text { eyx })^{2}=$ eyx $($ strongly $E$ reflexive inverse semigroups, see [7]).

For a given congruence $\rho$ we may ask for the least congruence containing $\rho$ and having one of the properties characterizing the congruences in the diagram. For $\sigma, \eta$ and $\nu$ this is simply the supremum $\sigma \vee \rho, \eta \vee \rho$ and $\nu \vee \rho$ since three congruences represent varieties. We give below an expression for the least $E$-unitary congruence containing $\rho$ (its existence was established in the proof of $[9$, Theorem 6.1]).

Proposition 6.5. For any congruence $\rho$ on $S$,

$$
\pi_{\rho}=(\sigma \rho \sigma \cap \rho \mathfrak{L})^{*}=((\rho \vee \sigma) \cap \mathcal{L} \rho \mathcal{L})^{*}
$$

is the least E-unitary congruence containing $\rho$.

Proof. In view of the correspondence of congruences on $S$ containing $\rho$ and the congruences on $S / \rho$, we conclude that the least $E$-unitary congruence on $S$ containing $\rho$ is given by

$$
a \pi_{\rho} b \Leftrightarrow a \rho \pi_{S / \rho} b \rho .
$$

Recall from Remarks 6.3 that $\pi=(\sigma \cap \mathcal{L})^{*}$. It thus suffices to prove

$$
a\left(\sigma \rho \sigma \cap \mathcal{L}_{\rho} \mathcal{L}\right)^{*} b \Leftrightarrow a \rho\left(\sigma_{S / \rho} \cap \mathcal{L}_{S / \rho}\right) * b \rho
$$

and for this, it is enough to show

$$
a\left(\sigma \rho \sigma \cap \mathcal{L}_{\rho} \rho\right) b \Leftrightarrow a \rho\left(\sigma_{S / \rho} \cap \mathcal{E}_{S / \rho}\right) b \rho .
$$

For this, it is further sufficient to show the equivalences

$$
\begin{aligned}
& a \sigma \rho \sigma b \Leftrightarrow a \rho \sigma_{S / \rho} b \rho, \\
& a \varrho \rho \in b \Leftrightarrow a \rho \varrho_{S / \rho} b \rho .
\end{aligned}
$$

Let $a \sigma \rho \sigma b$. Then $a \sigma x \rho y \sigma b$ for some $x, y \in S$, whence $a e=x e, x \rho y, y f=b f$ for some $e, f \in E$. Hence $a g \rho b g$, where $g=e f$ so that $(a \rho)(g \rho)=(b \rho)(g \rho)$, where $g \rho \in E_{S / \rho}$ and, thus, $a \rho \sigma_{S / \rho} b \rho$.

Conversely, let $a \rho \sigma_{S / \rho} b \rho$. Then for some $g \in E$, we have $(a \rho)(g \rho)=(b \rho)(g \rho)$ so that $a g \rho b g$. It follows that $a \sigma a g \rho b g \sigma b$ and thus $a \sigma \rho \sigma b$. This verifies (1). Formula (2) is verified similarly.

\section{REFERENCES}

1. D. G. Green, The lattice of congruences on an inverse semigroup, Pacific J. Math. 57 (1975), 141-152.

2. J. M. Howic, An introduction to semigroup theory, Academic Press, New York, 1976.

3. , The maximum idempotent separating congruence on an inverse semigroup, Proc. Edinburgh Math. Soc. 14 (1964), 71-79.

4. J. M. Howic and (3. Lallement, Certain fundamental congruences on a regular semigroup. Proc. Glasgow Math. Assoc. 7 (1966), 145-156.

5. D. B. McAlister, E-unitary inverse semigroups over semilattices, Glasgow Math. J. 19 (1978), 1-12. 
6. L. O'Carroll, Reduced inverse and partially ordered semigroups, J. London Math. Soc. 9 (1974), 293-301.

7. __ Strongly E-reflexive inverse semigroups, Proc. Edinburgh Math. Soc. 20 (1976-77), $339-354$

8. M. Petrich, Congruences on inverse semigroups, J. Algebra 55 (1978), 231-256.

9. N. R. Reilly and W. D. Munn, E-unitary congruences on inverse semigroups, Glasgow Math. J. 17 (1976), 57-75.

10. N. R. Reilly and H. E. Scheiblich, Congruences on regular semigroups, Pacific J. Math. 23 (1967), 349-360.

11. H. E. Scheiblich, Kernels of inverse semigroup homomorphisms, J. Austral. Math. Soc. Ser. A 18 (1974), 289-292.

Department of Mathematics, Simon Fraser University, Burnaby, British Columbia, Canada V5A 1 S6 